\title{
The adaptation to standing long jump distance in parkour is performed by the modulation of specific variables prior and during take-off
}

\author{
Sidney Grosprêtre ${ }^{1, *}$, Pierre Ufland ${ }^{2}$, and Daniel Jecker ${ }^{3}$ \\ ${ }^{1}$ EA4660-C3S Laboratory - Culture, Sport, Health Society, UPFR Sport, Besançon, France \\ 2 Institute of Physiology-Mitochondria, Oxidative Stress and Muscle Protection, Faculté de Médecine, Institut de Physiologie, \\ Strasbourg, France \\ 3 Laboratory "Sport and Social Sciences" (E3S), Faculté des Sciences du Sport, Strasbourg, France
}

Received 7 April 2017, Accepted 15 October 2017

\begin{abstract}
The present study aimed at investigating different variables that can be manipulated prior to and during take-off, to execute a specific standing long jump (SLJ) distance, according to jump expertise in parkour practitioners (=traceurs). Fourteen healthy young traceurs were included and separated into two groups: beginners (BEG) and experts (EXP). Firstly, classical vertical jump battery was used to characterize participants arm use and leg efficiency. Secondly, standing long jump (SLJ) performances were analyzed at four distances: 70, 80, 90, and 100\% of each participant's maximal SLJ distance. The force-time curves of the ground reaction forces $(\mathrm{GRF})$ and the center of pressure $(\mathrm{CoP})$ trajectory were measured with a force platform during the jump impulses. Take-off speed, angle and jump trajectory were estimated. For all of the participants, takeoff speed and angle, power output, and vertical GRF during jump preparation (counter movement) varied with distance. The EXP group exhibited greater backward CoP excursion, greater arm participation, greater take-off velocity and a greater modulation of take-off angle than BEG group. When comparing jumps of similar distance, EXP exhibited a more curvilinear trajectory with a higher peak than BEG. To conclude, different motor strategies can be adopted based on the jump distance, and these strategies can evolve as parkour experience increases.
\end{abstract}

Keywords: traceurs, center of pressure, ground reaction force, squat jump, counter movement jump

Résumé- La modulation de variables spécifiques permet de gérer la distance du saut en longueur sans élan en parkour. La présente étude a pour objectif d'analyser les différentes variables qui peuvent être modulées avant et pendant le décollage afin de réaliser un saut en longueur sans élan à une distance donnée, en fonction de l'expertise de pratiquants de parkour (= traceurs). Quatorze jeunes traceurs ont été inclus et divisés en deux groupes: les débutants (BEG) et les experts (EXP). Premièrement, une batterie de sauts verticaux classiques a été utilisée pour caractériser l'efficacité élastique ou l'utilisation des bras des deux groupes de participants. Deuxièmement, des sauts en longueur sans élan (SLJ) ont été exécutés à quatre distances : 70, 80, 90 et $100 \%$ de la distance maximale de chaque participant. La courbe force-temps de réaction au sol (GRF), les déplacements du centre de pression ( $\mathrm{CoP}$ ) ont été mesurés pendant l'impulsion et la préparation des sauts grâce à une plateforme de force. La vitesse et l'angle de décollage, ainsi que la trajectoire aérienne des sauts a été estimés grâce à ces données. Pour tous les participants, la vitesse de décollage, la puissance développée et la GRF pendant la préparation du saut (contre-mouvement) a été modulée en fonction de la distance du saut. Le groupe EXP a montré une plus grande excursion arrière du CoP, un index de participation des bras plus élevé et une meilleure modulation des angles de départ en comparaison au groupe BEG. En comparant des sauts à distance égale, une trajectoire plus courbe avec une hauteur plus élevée a été observée pour les EXP. Pour conclure, des stratégies motrices différentes peuvent être adoptées en fonction de la distance du saut, et ces stratégies évoluent avec l'expérience en parkour.

Mots clés : traceurs, centre de pression, force de réaction au sol, squat jump, counter movement jump

\footnotetext{
*Corresponding author: sidney.grospretre@univ-fcomte.fr
} 


\section{Introduction}

From a projectile perspective, launching an object for an accurate distance rather than for a maximum distance necessitates combining several factors to provide the best trajectory, i.e., to modulate the final projection angle and take-off velocity (Dupuy, Mottet, \& Ripoll, 2000). Then, when referring to whole-body movements such as sub-maximal horizontal jumps, one would question how an athlete can modulate such take-off characteristics when the goal is to reach a specific distance rather than executing the maximal performance. It has been argued that the length of an horizontal jump was mostly determined by the phases preceding take-off rather than by its maximal power output at take-off (Ruddock \& Winter, 2016). However, to date, there is no consensus regarding which variables accurately predict jumping distance in horizontal jumps, i.e., allow an athlete to modulate his take-off characteristics to land at an accurate distance.

Managing jump take-off characteristics to provide the optimal trajectory and then reach a pre-determined distance can be of major importance in some specific activities. Indeed, contrary to track and field jumping disciplines, some other sport activities require to jump accurately and not necessarily as far as possible. Among them, parkour is an activity in which jump accuracy is one of the most important skills to develop with training. Parkour is a discipline formally developed in France which is generally practiced in an urban environment but can be practiced in every landscape. This discipline is based on the use of urban (walls, barriers, etc.) or natural elements (trees, rocks, etc.) as obstacles. Thus, the main goal is to overcome these obstacles as efficiently as possible without external assistance equipment. Most of the jumps are performed with the constraint of executing an accurate distance, such as from the edge of one wall to the other. In this case, the specificity also arises from the lack of a run-up. Thus, traceurs are particularly familiar with the standing long jump (SLJ) - a horizontal jump performed with both feet simultaneously without a runup. Indeed, it was previously shown that parkour practitioners (= traceurs) could develop particular skills compared to other jumping disciplines such as gymnastics or track and field athlete, particularly in SLJ performance (Grosprêtre \& Lepers, 2015).

Some previous studies of the SLJ, although not parkourspecific, have shown that prior to and during the jump, the athlete has to exert the high mechanical power of the lower joints associated with an optimal take-off angle (Graham-Smith \& Lees, 2005; Wakai \& Linthorne, 2005). Indeed, it has been shown that low take-off angles (between 20 and $30^{\circ}$ ) resulted in longer SLJ distances (Wakai \& Linthorne, 2005). Superior performances in the SLJ were also shown to be associated with a high upper-tolower limb coordination, i.e., a great synchronization of arm and leg actions (Ashby \& Delp, 2006). However, it is not currently known which variable can be modulated if jump performance is accompanied by the constraint of having to reach a specific distance, nor if the strategies to manage such distance accuracy can evolve with training.

The present study investigated how traceurs change the mechanics of the movement (e.g., magnitude or direction) to produce the desired change in the projection variable(s) in order to perform an accurate horizontal jump performance with the take-off and landing at ground level. Since the few previous studies performed on traceurs' biomechanics have showed specific postural control and lower vertical force during landing (Maldonado, Bitard, Watier, \& Soueres, 2015; Puddle \& Maulder, 2013) this study will also focus on these two aspects, by analyzing both ground reaction force characteristics and center of pressure trajectories. From these aspects, it is therefore possible to determine take-off characteristics (angle, velocity) and finally to estimate the trajectory of the jumps. Beginners and expert traceurs were recruited in order to examine how parkour experience impacts such jumping skills. Assessing variables such as arm participation, classic vertical counter movement jumps, and squat jump performance would also help determine which parameters evolved with an athlete's expertise and were linked with the ability to jump accurately. These measurements helped to determine the following: - if performing SLJs of increasing distances results in similar jump impulse patterns that are simply amplified, or if selected variables may be modulated, thereby inducing different patterns for different distances;

- if this scheme is modified as parkour experience increases.

The present study aims to bring new insight into the understanding of jump motor control to determine which variables improve performance. It can be hypothesized that the specific variables that are manipulated both during jump preparation and take-off to perform an accurate jump distance differ from parkour beginners to experts.

\section{Material and methods}

\subsection{Participants}

Fourteen healthy young males (age: $22.1 \pm 3.8$ years, height: $175.6 \pm 1.5 \mathrm{~cm}$, weight: $67.2 \pm 4.2 \mathrm{~kg}$ ) gave informed consent to participate in the study. All were traceurs and regularly practiced parkour. They completed a form indicating experience, training habits, and training frequency.

Participants were subdivided into two groups pursuant to their experience in parkour: parkour beginners $(\mathrm{BEG}, \mathrm{n}=7)$ and parkour experts (EXP, $\mathrm{n}=7$ ). The beginners group (BEG) had been involved in parkour for less than 1 year (mean: $0.6 \pm 0.3$ years) at a regular practice ( 2 to 6 hours per week). The expert group (EXP) included participants with at least 3 years of regular experience in parkour (mean: $5.5 \pm 1.6$ years) that practice for 4 or 5 days per week (for a total of $10 \mathrm{~h}$ per week). Participants were all asked to wear crosstraining shoes to avoid excessive inter-participants 
variability. Indeed, barefoot or minimalist were not allowed to avoid footwear effect on ground reaction force (Harry et al., 2015).

All examinations were performed during the participants' normal training program. Written informed consent was provided by the participants. They were fully advised of the procedures and free to stop the experiment at any time. They had no history or clinical signs of neurological or physical disorders. Participants were not currently taking prescribed medications. All of the experimental procedures were performed in agreement with the Declaration of Helsinki and approved by the local Ethics Committee of the University.

\subsection{Overview of the experimental design}

Athlete's performance was qualitatively tested on one single experimental session. Before the experimental session, the participants were familiarized with the testing procedures and were told the following: not to perform exercises on the day before the test. Prior to data collection, the participants were instructed to perform a ten-minute warm-up that included jogging at a comfortable pace. The testing procedure consisted of executing several different jumps on a force platform. A two-minute passive recovery period was allowed between each jump.

\subsubsection{Horizontal jumps}

Participants were asked to perform SLJs for several distances of $70,80,90$ and $100 \%$ of the maximal distance that they could reach. The submaximal jumps were performed in a random order.

\subsubsection{Assessment of jumping indexes}

To estimate the athlete's use of stretch-shortening cycle and arm swinging, classic indexes were determined from the horizontal and vertical jumps. The participants performed maximal SLJs without arm swing by placing their hands on their hips. Two types of maximal vertical jumps were performed: squat jumps (SJ) and counter movement jumps (CMJ). Both were also performed with and without arm swing.

\subsection{The force platform}

All jumps were performed on a portable force plate (AccuPower; AMTI, Watertown, MA) with the dimensions $76 \times 102 \times 12 \mathrm{~cm}$ (length $\times$ width $\times$ height) which was positioned on a concrete floor. The force platform was secured on the floor in order to prevent any small slipping movements backward during horizontal jump impulses. Data were collected with the system sampling at $1000 \mathrm{~Hz}$. Force-time traces were continuously recorded during each condition saved with computer software (AccuPower Software v.1.0, Frappier Acceleration Sports Training, Fargo, ND) for further analysis. Data such as force (x, y, z axis), total power output, center of pressure position ( $\mathrm{x}, \mathrm{y}$ axis) and ellipse, were directly available through the software.

\subsection{Horizontal jumps}

Unlike vertical jumps, during SLJs, the participants did not land on the force platform. A landing platform was emulated by utilizing a gymnastics plinth with the dimensions of $160 \times 40 \times 12 \mathrm{~cm}$ (length $\times$ width $\times$ height) and placed at the desired distance. The height of the landing platform was carefully set to correspond with the force platform. Before each jump, participants were asked to observe one second of quiet standing, in order to set initial values of forces and velocity for further data analysis. Each jump was validated when the participant landed forefoot on an area materialized on the landing platform and maintained equilibrium for two seconds after landing. The number of successful trials over the total number of performed jumps was used to estimate the jump accuracy (\% of successful trials over the total number of trials).

At least two maximal SLJs were performed by the participants, and further trials were added until performance no longer increased. The maximal metered performance was measured with the consideration of the front edge of the force platform to the rear part of the most indented heel. Participants had to perform maximal performances with and without arm swinging. For this latter condition, they were asked to place their hands on the hip. Then, from the maximal metered performance with arms, submaximal distances (i.e., 70, 80, and $90 \%$ of the maximal SLJ measured length) were calculated. Afterward, participants had to successively execute these established distances from the force platform and manage their landing on the edge of the landing platform.

\subsection{Vertical jumps}

Participants were asked to jump and land with both feet simultaneously on the force platform, with no initial steps or shuffling. Angles of the knee and ankle were visually controlled during all landings. The SJ was assessed from a starting position with knees flexed at $90^{\circ}$ and weight well distributed over both feet. Participants were asked to keep their trunk straight, and no counter movement with the legs was allowed. For the CMJ performances, participants began in a standing upright position. They were asked to bend to $90^{\circ}$ knee flexion and immediately jump without pausing in the squat position.

\subsection{Data analysis}

\subsubsection{Jumping indexes}

The dependent variable for the CMJs and SJs was jump height. Jump height was directly obtained in the software, derived from the flight time. During the vertical jumps, CMJ and SJ heights allowed an effective estimation of the leg stretch-shortening cycle by calculating the Elasticity Index (Hara, Shibayama, Takeshita, Hay, \& Fukashiro, 2008). 
The Elasticity Index (EI) is a reliable index of elastic tissue performance during counter movement jumps (McGuigan et al., 2006). The EI is determined as the increase in performance by the use of counter-movement, using the following formula: $\mathrm{EI}=[(\mathrm{CMJ}-\mathrm{SJ}) / \mathrm{SJ}] \times 100$. To characterize the increase of performance with arm usage, an Arm Participation Index (API) (Borràs, Bsalius, Drobnic, \& Galilea, 2011) was quantified for each jump using the jump height with and without arm swing with the formula: API $=[(\mathrm{CMJa}-\mathrm{CMJs}) / \mathrm{CMJs}] \times 100($ e.g., for arm participation in CMJ, CMJa and CMJs corresponding to the jump with and without arms, respectively). The same calculation was performed to assess API in SJs and SLJs.

\subsubsection{Horizontal jumps}

The characteristics of the ground reaction forces (GRF) were assessed by analyzing the force-time curve obtained from the force platform. Maximal resultant force over the three axis ( $\mathrm{x}, \mathrm{y}, \mathrm{z})$ was determined and used as the maximal GRF. Maximal power output was calculated as the product of maximal GRF and velocity. A one-second quiet standing period before the jump preparation was used to set the initial value of the several variables. For instance, the total ground reaction force (GRF) was taken during this period to obtain participants' body weight (BW) and obtain their mass by dividing the weight by the gravitational acceleration (Owen, Watkins, Kilduff, Bevan, \& Bennett, 2014).

Firstly, to account for the differences between the participants' vertical and forward forces, the vertical and antero-posterior GRF were analyzed separately. Contrary to the classical analysis of the rate of force development during jump impulse, here the timing and force characteristics were separately analyzed to account for differences between these two aspects. Figure 1 summarizes the analysis performed on these GRF components. Vertical GRF was the first variable of interest, as it allows several stages of the jump impulse to be assessed. The minimal peak force value, under BW, accounted for the jump preparation phase. In order to separate timing and force characteristics, the maximal vertical GRF was measured and the time between the minimum and maximal vertical GRF $\left(\Delta_{T}\right)$, which characterizes the ability to increase force in order to reach the maximum value, was determined. Then, the peak of maximal force was also measured for antero-posterior GRF. Lateromedial GRFs were also controlled but negligible as the mean variation was lower than $0.5 \%$ of BW. In fact, the data showed that the SLJ can be considered and studied from the sagittal plane because the action of both limbs is symmetrical (Hickox, Ashby, \& Alderink, 2016).

Secondly, participant's weight was subtracted from the GRF to obtain the net force-time curve that was divided by the mass to obtain the acceleration (Newton's second law) and integrated to obtain the velocity of the system center of mass. The maximal velocity at take-off was then taken for analyzis (referred hereafter as "take-off speed"). By analyzing separately the horizontal and
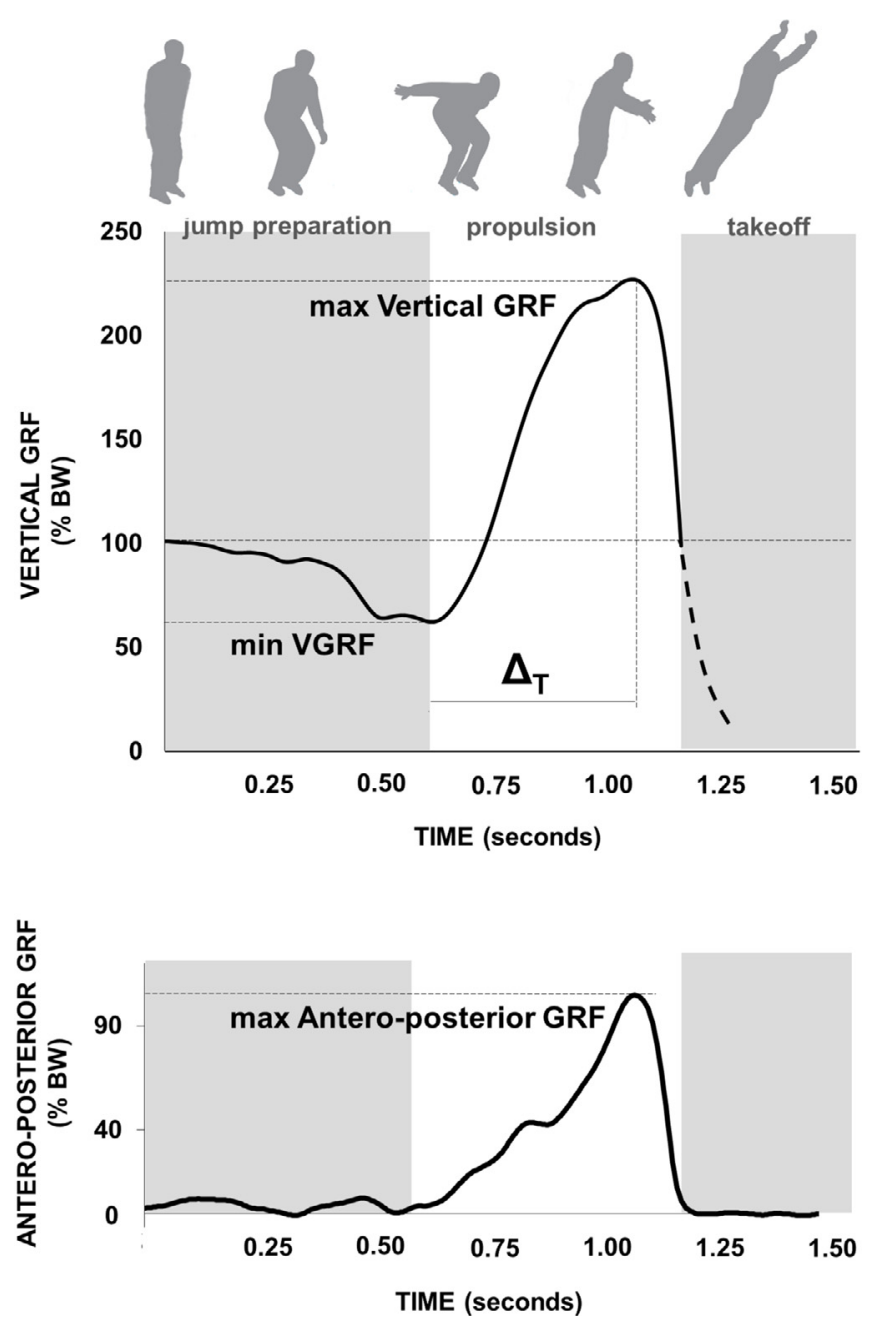

Figure 1. Peak analysis of the ground reaction force during the standing long jump impulse of one representative participant. In the upper panels, drawings of the different positions during different jump phases. GRF: ground reaction force. BW: body weight.

vertical components of the force-time curve, it was therefore possible to obtain the projection angle at takeoff. Finally, an estimation of the jump aerial trajectory was performed for each participant and SLJ with respect to the calculated take-off characteristics (speed and projection angle) and to the jump distance, considering that the trajectory is a parabolic curve.

Finally, the center of pressure $(\mathrm{CoP})$ displacement characteristics were analyzed. The $90 \%$ confidence ellipse was directly calculated by the software in the one-second quiet standing period before the jump. The CoP backward excursion before the take-off thrust was measured as follows: the maximal CoP excursion on the anteroposterior direction from the center of the ellipse of CoP displacements during the normal stance.

To account for the inter-group difference without the effect of jumping distance, for each participant a single sub-maximal jump of a given distance was extracted from all succeeded trials. Given the difference in jump capacity, 
this jump was necessarily at a higher percentage of maximal SLJ for BEG than for EXP. A single analysis was performed with all these variables on this performance.

\subsection{Statistical analysis}

All data are expressed as the mean \pm standard deviation. First, the normality of the data was verified by the Shapiro-Wilk test to ensure that classic analysis of variance (ANOVA) could be used.

A two-way ANOVA with repeated measures was performed on the jumping indexes for the factors of jump type (CMJ, SJ, SLJ) and group (EXP, BEG). For horizontal jumps, a two-way ANOVA with repeated measures was performed for the factors group (BEG, EXP) and jump distance (70, 80, 90, $100 \%$ of maximal SLJ distance) for all of jump parameters (GRFs, speed, power, accuracy, angle, and CoP excursion). When a main effect or interaction was found, a post hoc analysis was performed using an HSD Tukey's test. Group-related differences for all measurements regarding the analysis of similar-length jumps were analyzed with Student unpaired $t$ tests.

Statistical analysis was performed using STATISTICA software (8.0 version, Statsoft, Tulsa, Oklahoma, USA). The level of significance was set at $P \leq 0.05$.

\section{Results}

\subsection{Global analysis}

The distance $(P<0.001)$ and group $(P=0.043)$ factors were significant for the maximal power output developed in an SLJ (Fig. 2A). The EXP group showed greater power modulation among the various distances than the BEG group, who only showed significant power modulation between the maximal $(100 \%)$ and other submaximal (70, 80 and 90\%) distances (Fig. 2A). The jump accuracy was higher as the distance increased, and significantly greater for EXP compared to BEG at each distance of the SLJ (Fig. 2D). Contrary to the vertical jumps, both groups had significantly lower maximal SLJ performances without arm swing than with arm swing $(P<0.001)$.

\subsection{Vertical and horizontal jumping indexes}

The group factor was significant for the measurements of vertical jump height $(P=0.002)$ and power $(P=0.023)$; the EXP group outperformed the BEG group. Then, contrary to the BEGs, the EXPs showed a significant increment of performance during the vertical jump with arm swing. However, the group and arms factors had no effect on the force developed during vertical jumps ( $P=0.14$ and $P=0.45$, respectively). The EI (on average $12.65 \pm 5.15 \%)$ did not show any significant differences between the groups $(P=0.13)$.

The jump type $(P<0.001)$ factor had a significant effect on arm participation. The API was greater in maximal SLJs than in vertical jumps $(P=0.004$ and
$P=0.013$ for SJs and CMJs, respectively). The CMJ API was $11.2 \pm 9.2 \%$ for BEGs and $18.8 \pm 2.2 \%$ for EXPs $(P=0.004)$. For SJ performances, arm participation was estimated at $2.1 \pm 13 \%$ and $17.6 \pm 4.9 \%$ respectively for BEGs and EXPs $(P=0.012)$. SLJ arm participation was $24.5 \pm 9.5 \%$ for BEGs and $31.1 \pm 9.6 \%$ for EXPs $(P=0.04)$. The raw maximal SLJ performance without arms was still greater in EXP than in BEG $(P=0.01)$.

\subsection{Ground reaction forces (GRF)}

Contrary to the maximal total force output, analyzing the antero-posterior and vertical components of the GRF separately revealed differences among the SLJ distances performed.

Indeed, an interaction effect was found between the group and distance factors $(P=0.003)$ for the maximal antero-posterior GRF. Post hoc analysis revealed that in the EXP group, the antero-posterior GRF increased significantly from $70 \%$ of maximal SLJ to $100 \%$ (respectively, $80.6 \pm 5.9 \%$ and $119.6 \pm 7.1 \%$ of BW, $P<0.001$ ). In this group, significant differences were also observed between all of the other submaximal SLJ distances; antero-posterior GRF increased in the following order: $70<80<90<100 \%$ of the maximal SLJ $(0.001<P$ $<0.044)$. A similar evolution of the antero-posterior GRF according to the SLJ distance was observed in BEG group, despite the fact that only the extreme value (lowest at $70 \%$ SLJ and greatest at 100\% SLJ) showed a significant difference $(P<0.001)$. Regardless of the distance, the antero-posterior GRF was greater in the EXP group (from $80.6 \pm 5.8 \%$ BW at $70 \%$ SLJ to $119.6 \pm 7.1 \% \mathrm{BW}$ at $100 \% \mathrm{SLJ})$ than in the BEG group (from $71.4 \pm 4.3 \% \mathrm{BW}$ to $98.2 \pm 5.7 \% \mathrm{BW}$ ).

For the vertical GRF, the distance $(P<0.001)$ factor was significant for the minimum peak of the vertical GRF (Fig. 3A). The minimum vertical GRF decreased as the distance increased; this effect was similar for both the BEG and EXP groups because the group factor did not affect the results $(P=0.69)$. The group and distance factors for $\Delta_{T}(P=0.02)$ showed significant interaction. Indeed, only the EXP group showed a significant decrease in $\Delta_{T}$ when the SLJ distance increased (Fig. 3C).

\subsection{Trajectories}

The factor group significantly affected the take-off speed $(P<0.001)$, but not the factor distance $(P=0.24)$, meaning that all groups showed a similar increase in the take-off speed according to the jump distance (Fig. 4A). A significant interaction between factors group and distance was found for the take-off angle $(P=0.04)$. Only the EXP group exhibited a significant modulation of the take-off angle, which was increased according to the distance of the jump (Fig. 4B). Fig. 4C depicts the estimated trajectories of the jumps for both groups according to the take-off parameters and to the distance measured. 

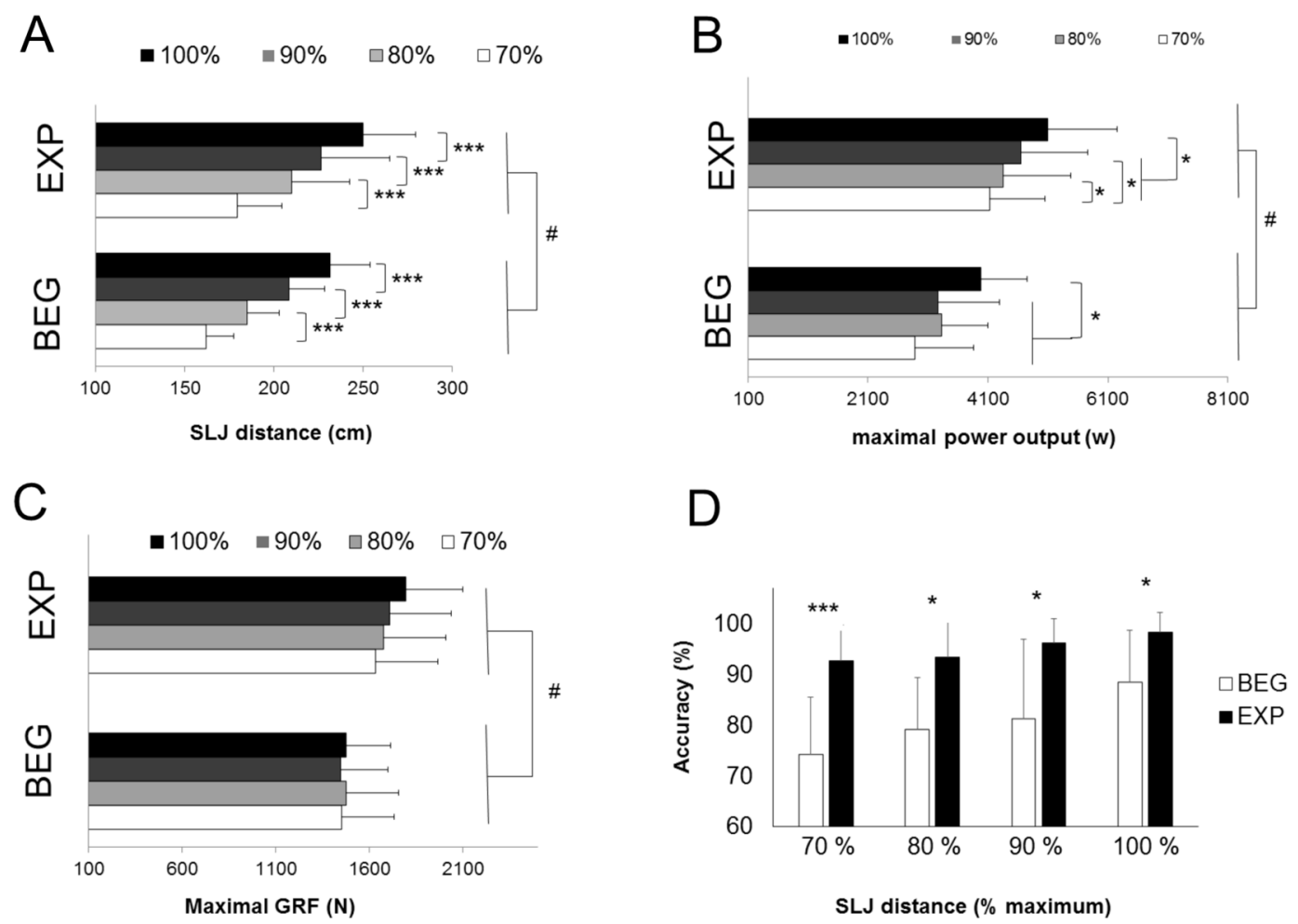

Figure 2. General standing long jump (SLJ) characteristics. The percentages represent maximal (100\%) and sub-maximal (70, 80, $90 \%$ ) antero-posterior jumping distances. A. Distances $(\mathrm{cm})$ performed by parkour experts (EXP) and beginners (BEG). B. Maximal take-off power (W) relative to SLJ distance. C. Maximal ground reaction force (GRF) of jumps. D. Accuracy of each group at the four distances. Accuracy represents the percentage of the number of successful jumps over the total number of performed jumps. ${ }^{*},{ }^{* * *}$ : significant intra-group differences with $P<0.05$ and $P<0.001$, respectively.

\subsection{CoP analysis}

Concerning backward excursion of the CoP before take-off, only the 100\% SLJ (Fig. 5) showed a significant difference between the groups. At this distance, the EXP group exhibited greater excursion $(P<0.001)$ than the BEG group (Fig. 5). Contrary to the BEG group, the EXP group showed a significant difference $(P$ $<0.001$ ) between backward excursion at $100 \%$ SLJ distance and backward excursion at the other distances (Fig. 5).

\subsection{Same-distance comparison}

To assess the motor strategies at a comparable performance, a SLJ distance of similar length was taken for analysis among all participants. Naturally, this matched distance between BEG $(196.6 \pm 2.7 \mathrm{~cm})$ and EXP $(196.1 \pm 3.6 \mathrm{~cm})$ represented a higher percentage of the maximal performance $(P=0.02)$ for BEG $(85.7 \pm 3.7 \%$ of maximal SLJ) than for EXP $(75.0 \pm 3.2 \%)$. The jump accuracy was greater for EXP $(93.6 \pm 4.7 \%)$ than for BEG $(75.0 \pm 11.2 \%)$. Regarding jump preparation, backward $\mathrm{CoP}$ excursion and the minimal vertical GRF were statistically greater in EXP compared to BEG $(P=0.02$ and $P=0.03$, respectively).

Interestingly, the take-off speed was statistically greater $(P=0.04)$ in EXP than in BEG, as well as the take-off angle, leading the trajectory determination to exhibit different profiles. In fact, at this given distance, EXP exhibited a higher peak of the estimated curve than BEG (Fig. 6).

\section{Discussion}

The aim of the present study was to analyze different variables that appear to be modulated during the jump impulse to achieve an accurate SLJ distance according to parkour expertise. For that purpose, the impulse characteristics of horizontal jumps at several distances, as well as the jumping skills for BEG and EXP groups of parkour athletes (=traceurs) were assessed. The results were as follows:

- jump accuracy and power output, but not resultant force, are differently modulated between EXP and BEG according to the jump distance;

- force-time curve characteristics were modulated in line with jump distance and expertise in parkour, especially in the phases that preceed take-off;

- EXP group, with lower take-off angles and higher takeoff speed, showed different jump trajectory profiles than BEG.

As expected, EXP group exhibited greater maximal jump performances and developed higher power output than BEG, emphasizing that parkour training enhances 

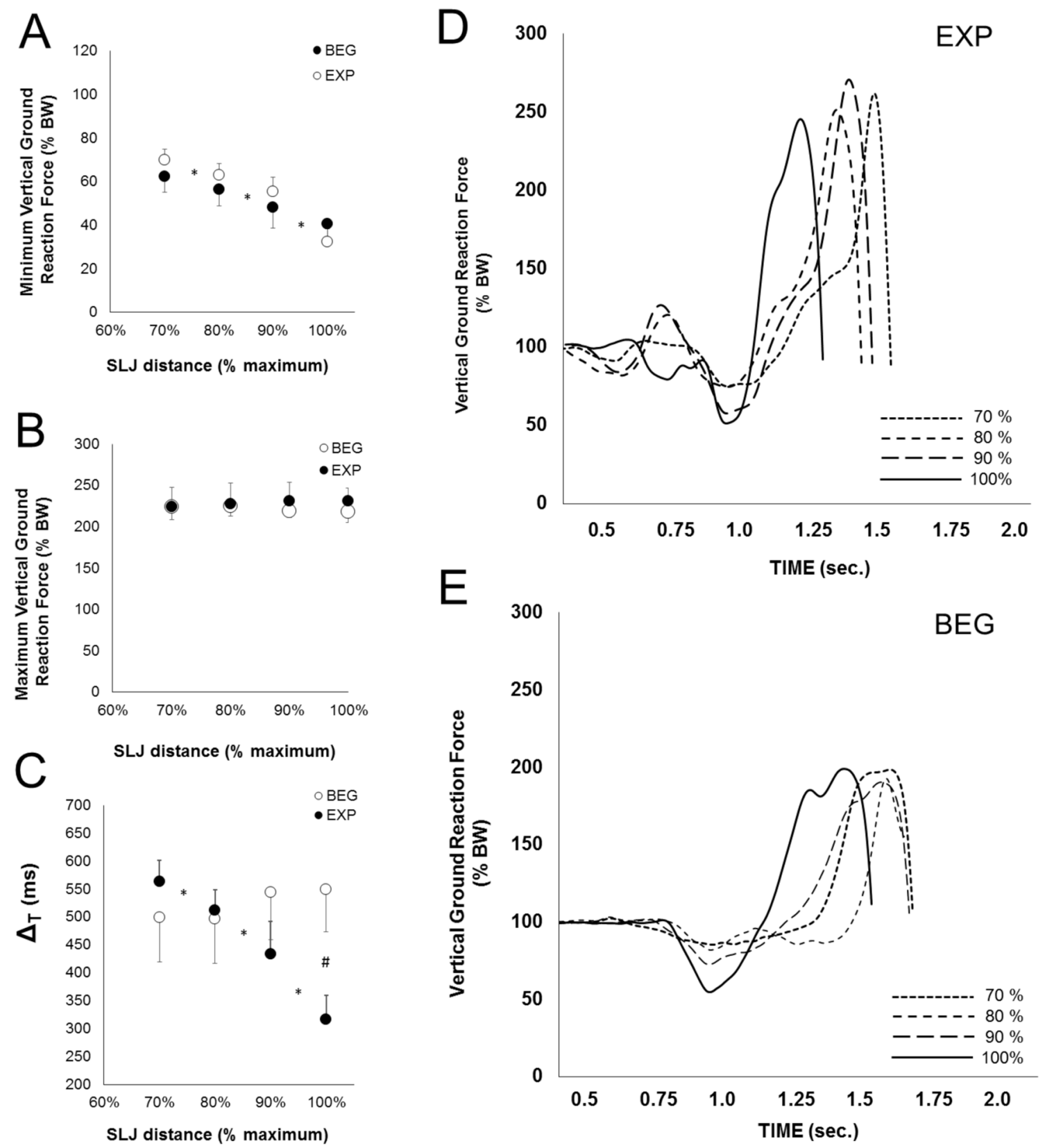

Figure 3. Vertical ground reaction force during horizontal jumps at several distances. Temporal and magnitude specificity of the vertical ground reaction forces are depicted for the parkour beginner group (BEG, black round and Figure E) and expert group (EXP, white rounds and Figure D), for the four distances tested (from 70 to $100 \%$ of maximal SLJ performance). A. Peak of minimal vertical ground reaction force expressed in \% of body weight (BW). B. Peak of maximal vertical ground reaction force expressed in \% of BW. C. Peak-to-peak delta of time between minimal and maximal peaks in milliseconds. D. and E. represent recordings of two representative participants, respectively from the EXP and BEG groups. Vertical ground reaction forces are depicted as a solid line for the $100 \%$, large dashes for $90 \%$, small dashes for $80 \%$ and dots for $70 \%$ distances. ${ }^{*}$ : significant inter-distance difference $(P<0.05)$. \# : significant intergroup difference $(P<0.05)$.

both horizontal and vertical jump performances (Grosprêtre \& Lepers, 2016). The present study also showed that parkour experience could lead to a better jump accuracy, i.e., a better percentage of successful trials, emphasizing a better motor control. Particularly, in the BEG group the accuracy was decreased as the distance was small, meaning that parkour training, in addition to lead athletes to develop higher maximal performance, also leads to a more accurate gradation of sub-maximal performances.

It has been previously noted that parkour landing can lead to a vertical GRF ranging from 2.5 to $3.6 \mathrm{BW}$ when the drop jump is performed from $50 \%$ of body height (Standing \& Maulder, 2015). The present study demonstrated that the jump impulse in parkour can also lead to great vertical GRF in maximal SLJ (around 2.5 BW), 


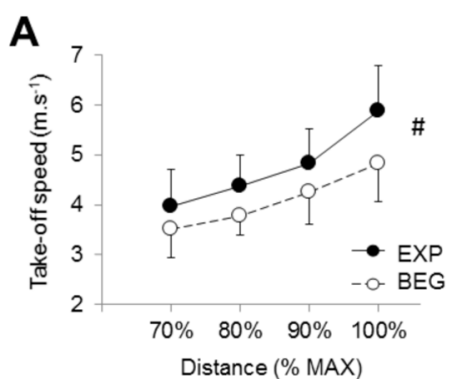

B

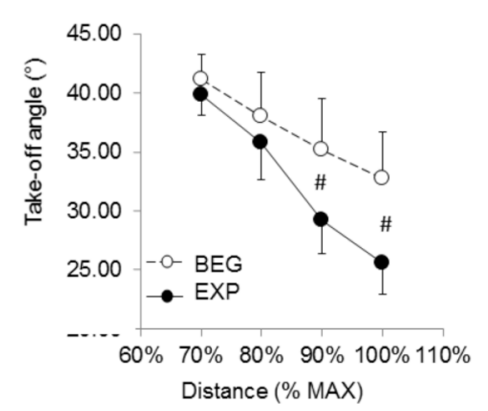

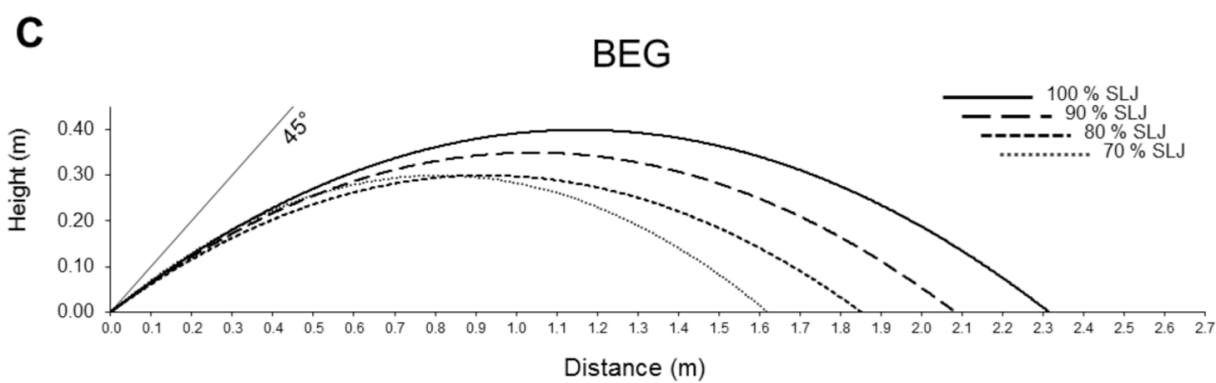

EXP

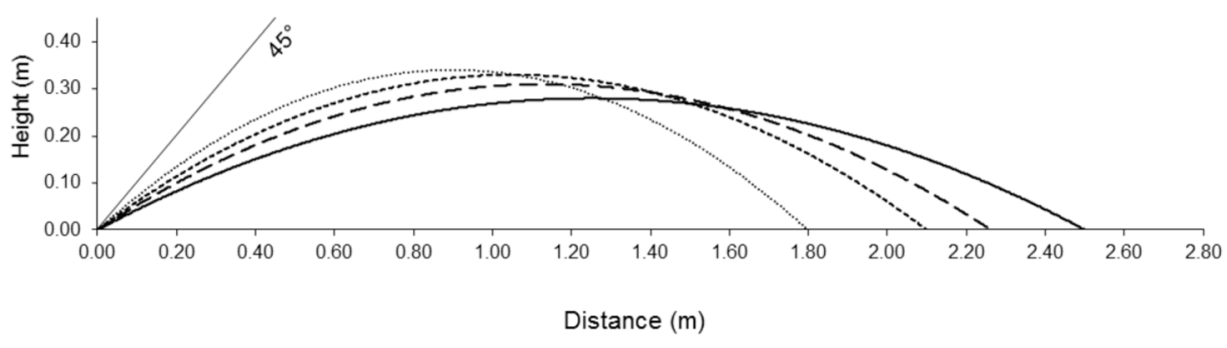

Figure 4. Trajectory determination for EXP and BEG at the several distance. A. Take-off speed for both groups at $70,80,90$ and $100 \%$ of maximal standing long jump (SLJ) distance. B. Take-off angle for both groups at 70, 80, 90 and $100 \%$ of maximal SLJ distance. C. Estimated trajectories for each distance for the BEG group (upper panel) and the EXP group (lower panel). \#: significance intergroup difference $(P<0.05)$.

which did not differ significantly between BEGs and EXPs. However, no significant maximal or vertical GRF modulation was observed in the various distances, meaning that these variables do not seem to be a factor that impacts the ability to perform a distance adjustment in an SLJ, for BEGs as well as for EXPs. Indeed, despite an increase in maximal force production, strength training does not necessarily modify the accuracy of force gradation (Smits-Engelsman, Smits, Oomen, \& Duysens, 2008). It was shown that peripheral characteristics, such as longer arms and legs or muscle capacity, might not influence jump performances in adults (Hraski, Hraski, Mrakovi, \& Horvat, 2015; Wu, Wu, Lin, \& Wang, 2003). In the present study, parkour BEGs and EXPs achieved similar elasticity index, raising the fact that such peripheral characteristics may not be a factor that explains different jump performances with parkour expertise. This corroborates the fact that sub-maximal performance accuracy is more related to specific motor strategies than to the capacity to develop high muscle force and power.

On the contrary, this study showed that parkour BEGs have lower arm participation than EXPs in both vertical and horizontal jumps. It was already found that traceurs with more experience utilize greater arm participation to perform vertical jumps compared to non-trained athletes or athletes from other disciplines (Grosprêtre \& Lepers, 2015), APIs of EXPs being reported to reach $18 \%$ in vertical jumps (e.g., CMJ). These results, linked to CMJ performances, are identical to those of the EXP group in the present study $(\sim 18 \%)$. The present study adds the new fact that arm participation in horizontal jumps $(\sim 33.5 \%)$ was statistically greater than arm participation in vertical jumps for all participants, emphasizing the importance of arm swing in motor strategies of horizontal jump impulse. However, the fact that maximal SLJ without arms was still higher in EXP than in BEG showed that arms swinging is not the only variable that explain different performances with parkour expertise.

Contrary to the maximal GRF at take-off, the minimum peak of vertical GRF was modulated in line with jumping distance. This minimal vertical GRF, under BW, can be explained by the angular momentum created by counter movement generated by leg extension as well as backward arm swing. In addition, it was shown that backward arm swing was strongly related to a backward shift of the center of mass (Ashby \& Heegaard, 2002). Although the center of mass displacement is not directly linked with CoP trajectory during dynamic moves, this is consistent with the results found on $\mathrm{CoP}$ backward excursion. Indeed, EXP group showed a modulation of backward $\mathrm{CoP}$ excursion according to jump distance contrary to BEG group, indicating a greater postural sway with parkour experience. It has been reported that maximal SLJ performance requires a backward shift in the center of mass of $8 \mathrm{~cm}$ (Ashby \& Heegaard, 2002) or $7.5 \mathrm{~cm}$ (Wu et al., 2003). This unstable backward position has two main objectives (Wu et al., 2003):

- putting the athlete in a position that requires minimal force to more easily select the required direction;

- providing the maximal amplitude of arm and leg movements to generate the maximal forward momentum.

The modulation of these variables in the early phases of the jump preparation may allow the athletes to adjust the final take-off characteristics. Indeed, the estimated take- 
A

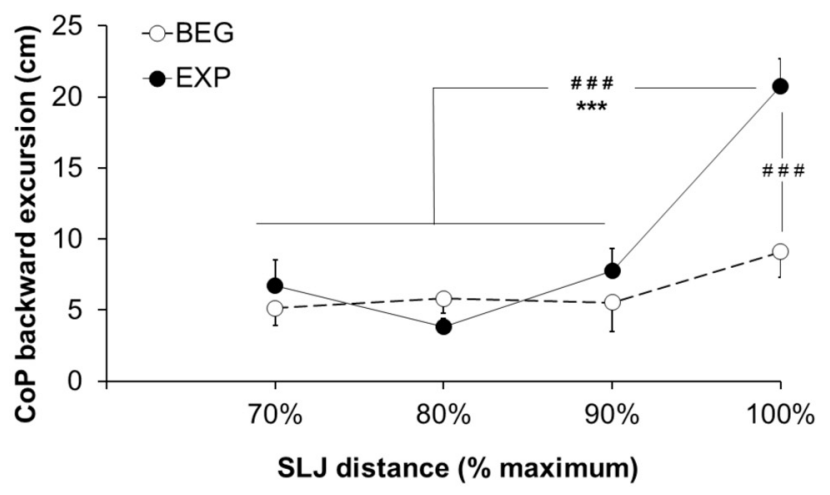

B

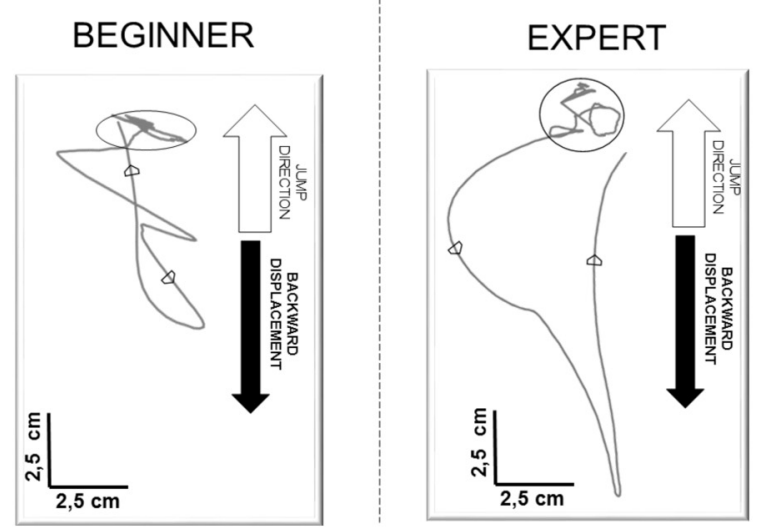

Figure 5. Center of pressure ( $\mathrm{CoP})$ displacement characteristics before take-off. A. CoP backward excursion before the SLJ take-off for the various distances. B. Representative CoP backward excursions of one parkour expert and one parkour beginner. The black circles delimit the ellipse of CoP excursion in the normal stance before take-off. Arrows indicate the direction of the CoP excursion. ${ }^{*},{ }^{* * *}$ : significant difference between the several conditions, at $P<0.05$ and $P<0.001$, respectively. \#\#\#: significant inter-group differences with $P<0.001$.

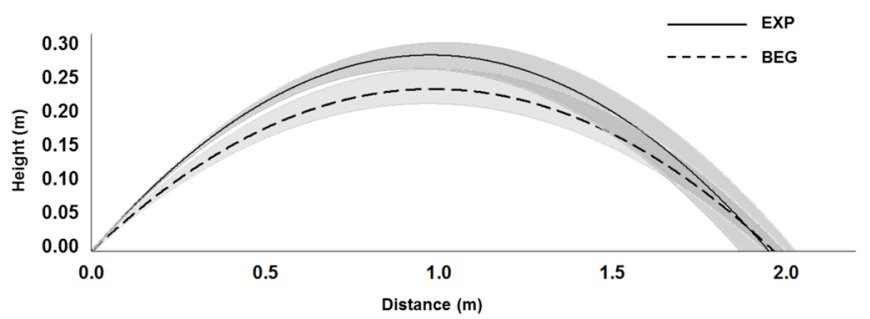

Figure 6. Estimated jump trajectory for BEG and EXP at the same distance. The grey areas represent the standard deviation of each trajectory.

off speed was also shown to be modulated according to jump distance. This velocity was increased from the smallest distance to the longest for both groups, although globally higher in EXP group. One of the clues to explain this inter-group difference can be found when analyzing the temporal characteristics of GRF. EXP group exhibited lower and better gradation of the time between the minimal and maximal vertical GRFs $\left(\Delta_{T}\right)$, characterizing the delay between counter movement and take-off thrust. This observation indicates that the timing characteristics of the movement, mostly modulated by the speed of execution, are important for SLJ performance modulation. The shorter $\Delta_{T}$ of EXP could arise from the shorter delay between the eccentric and concentric phases of the jump (Toumi, Best, Martin, F'Guyer, \& Poumarat, 2004). In fact, studies have shown that plyometric training such as parkour can improve the efficacy of the stretch-shortening cycle and then reduce this delay (Markovic, 2007; Turner, Owings, \& Schwane, 2003).

Concerning take-off characteristics, the present study also showed that the estimated take-off projection angle could be modulated according to the jump distance. Indeed, both groups exhibited a decrease of take-off angle from 70 to $100 \%$ of maximal SLJ. Nonetheless, EXP groups showed a higher range of angles and lower take-off angles at the greatest SLJ distances. In fact, it has been reported that the take-off angle for an SLJ should be minimized to increase jump distance; the optimal projection angle for maximal performance being lower than $45^{\circ}$, the theoretical angle to optimize a parabolic trajectory of a projectile. Wakai \& Linthorne in 2005 showed that physically active males, but not specifically trained to jumping disciplines, have a preferred angle of $\sim 34^{\circ}$ to perform a maximal SLJ, while a theoretical calculus showed that the optimal angle should be closed to $\sim 22.6^{\circ}$. The present study demonstrates that parkour training leads to an optimization of this variable, experienced traceurs showing low angles when performing maximal SLJ $\left(\sim 25.6^{\circ}\right)$ compared to beginners $(\sim 32.7)$. This result can be partly explained by the fact that EXPs are highly likely to be familiar with low take-off angles because the SLJ is a standard move in parkour. For non-parkour trained athletes or beginners, such low angles at take-off, meaning a high body tilt forward, may increase the risk of falling forward. This last consideration is linked with the fact that EXPs showed a greater backward CoP excursion at maximal distance, accounting for a greater total anteroposterior tilt during the jump impulse. A previous study showed that backward and upward CoP excursions during the jump impulse are strongly linked to control forward body disequilibrium (Le Pellec \& Maton, 2002). A strong link between balance and SLJ performance has already been found (Hammami, Chaouachi, Makhlouf, Granacher, \& Behm, 2016), implying a transfer effect from balance to power training and vice versa. Parkour involving both types of training, postural control specificity has already been noted for parkour landings compared with traditional landings (Maldonado et al., 2015). Then, the present results suggest that specific postural strategies also exist at take-off.

Finally, the specificity of these take-off characteristics between BEG and EXP leads in the present study to observe different theoretical parabolic trajectory of the aerial phase of the jumps. In fact, EXP groups showed a more curvilinear trajectory with higher peak at the smallest jump distances. This particular shape can be attributed to the requirement to land at a specific distance 
and to maintain the landing position without stepping forward. If the aerial trajectory is a symmetrical parabolic curve, one would consider that the greater take-off angle will also induce a greater landing angle. This leads to have a more vertical trajectory when reaching the targeted distance, while minimizing the horizontal speed at landing. This type of postural vertical strategy can generally be seen as the safest way to maintain the balance (Hilt, Berret, Papaxanthis, Stapley, \& Pozzo, 2016). When practicing parkour, not falling upward or backward when landing on a wall can be of particular importance when the jump is performed from a certain height.

To summarize, as expected from previous studies on parkour (Grosprêtre \& Lepers, 2015) or other disciplines such as trampoline (Aalizadeh, Mohammadzadeh, Khazani, \& Dadras, 2016), training had a significant impact on the maximal jump performances compared to non-trained individuals. The present study showed that parkour also leads to a better sub-maximal performance gradation. This was allowed by different jump strategies adopted by EXP compared to BEG, from the early stages of the jump preparation, as shown by the force-time curves' characteristics or backward CoP excursion, to the final take-off parameters. The use of kinematic measurements (e.g., by motion capture), can help to corroborate the different jump aerial trajectory that where estimated from the present data. To conclude, parkour training seems to enhance not only leg power but also the ability to control movement disequilibrium, have a better arm use and execute optimal take-off characteristics that contribute to accurate jumping distances and higher performance.

Acknowledgments. The authors are particularly grateful to the French Parkour Federation (Fédération de Parkour, FPK) for its help and support.

\section{References}

A. Dupuy, M., Mottet, D., \& Ripoll, H. (2000). The regulation of release parameters in underarm precision throwing. Journal of Sports Sciences, 18/6, 375-382. Available from https:// doi.org/10.1080/02640410050074304.

Aalizadeh, B., Mohammadzadeh, H., Khazani, A., \& Dadras, A. (2016). Effect of a trampoline exercise on the anthropometric measures and motor performance of adolescent students. International Journal of Preventive Medicine, 7/1, 91. Available from https://doi.org/10.4103/2008-7802.186225.

Ashby, B.M., \& Heegaard, J.H. (2002). Role of arm motion in the standing long jump. Journal of Biomechanics, 35/12, 1631-1637.

Ashby, B.M., \& Delp, S.L. (2006). Optimal control simulations reveal mechanisms by which arm movement improves standing long jump performance. Journal of Biomechanics, 39/9, 1726-1734. Available from https://doi.org/10.1016/j. jbiomech.2005.04.017.

Borràs, X., Balius, X., Drobnic, F., \& Galilea, P. (2011). Vertical jump assessment on volleyball: a follow-up of three seasons of a high-level volleyball team. Journal of Strength and Conditioning Research / National Strength \& Conditioning Association, 25/6, 1686-1694. Available from https://doi. org/10.1519/JSC.0b013e3181db9f2e.
Graham-Smith, P., \& Lees, A. (2005). A three-dimensional kinematic analysis of the long jump take-off. Journal of Sports Sciences, 23/9, 891-903. Available from https://doi.org/ $10.1080 / 02640410400022169$.

Grosprêtre, S., \& Lepers, R. (2015). Performance characteristics of Parkour practitioners: Who are the traceurs? European Journal of Sport Science, 1-10. Available from https:// doi.org/10.1080/17461391.2015.1060263.

Grosprêtre, S., \& Lepers, R. (2016). Performance characteristics of Parkour practitioners: Who are the traceurs? European Journal of Sport Science, 16/5, 526-535. Available from https://doi.org/10.1080/17461391.2015.1060263.

Hammami, R., Chaouachi, A., Makhlouf, I., Granacher, U., \& Behm, D.. (2016). Associations between balance and muscle strength, power performance in male youth athletes of different maturity status. Pediatric Exercise Science, 1-32. Available from https://doi.org/10.1123/pes.2015-0231.

Hara, M., Shibayama, A., Takeshita, D., Hay, D.C., \& Fukashiro, S. (2008). A comparison of the mechanical effect of arm swing and countermovement on the lower extremities in vertical jumping. Human Movement Science, 27/4, 636-648. Available from https://doi.org/10.1016/ j.humov.2008.04.001.

Harry, J.R., Paquette, M.R., Caia, J., Townsend, R.J., Weiss, L. W., \& Schilling, B.K. (2015). Effects of footwear condition on maximal jumping performance. Journal of Strength and Conditioning Research, 29/6, 1657-1665. Available from https://doi.org/10.1519/JSC.0000000000000813.

Hickox, L.J., Ashby, B.M., \& Alderink, G.J. (2016). Exploration of the validity of the two-dimensional sagittal plane assumption in modeling the standing long jump. Journal of Biomechanics, 49/7, 1085-1093. Available from https://doi. org/10.1016/j.jbiomech.2016.02.037.

Hilt, P.M., Berret, B., Papaxanthis, C., Stapley, P.J., \& Pozzo, T. (2016). Evidence for subjective values guiding posture and movement coordination in a free-endpoint whole-body reaching task. Scientific Reports, 6/1, 23868. Available from https://doi.org/10.1038/srep23868.

Hraski, M., Hraski, Z., Mrakovi, S., \& Horvat, V. (2015). Relation between anthropometric characteristics and kinematic parameters which influence standing long jump efficiency in boys and adolescents. Collegium Antropologicum, 39/Suppl 1, 47-55.

Le Pellec, A., \& Maton, B. (2002). Initiation of a vertical jump: the human body's upward propulsion depends on control of forward equilibrium. Neuroscience Letters, 323/3, 183-186.

Maldonado, G., Bitard, H., Watier, B., \& Soueres, P. (2015). Evidence of dynamic postural control performance in parkour landing. Computer Methods in Biomechanics and Biomedical Engineering, 18/Suppl 1, 1994-1995. Available from https:// doi.org/10.1080/10255842.2015.1069588.

Markovic, G. (2007). Does plyometric training improve vertical jump height? A meta-analytical review. British Journal of Sports Medicine, 41/6, 349-355; discussion 355. Available from https://doi.org/10.1136/bjsm.2007.035113.

McGuigan, M.R., Doyle, T.L.A., Newton, M., Edwards, D.J., Nimphius, S., \& Newton, R.U. (2006). Eccentric utilization ratio: effect of sport and phase of training. Journal of Strength and Conditioning Research / National Strength $\&$ Conditioning Association, 20/4, 992-995. Available from https://doi. org/10.1519/R-19165.1.

Owen, N.J., Watkins, J., Kilduff, L.P., Bevan, H.R., \& Bennett, M.A. (2014). Development of a criterion method to determine peak mechanical power output in a countermovement jump. Journal of Strength and Conditioning Research, 28/6, 1552-1558. Available from https://doi.org/10.1519/ JSC.0000000000000311. 
Puddle, D.L., \& Maulder, P.S. (2013). Ground reaction forces and loading rates associated with parkour and traditional drop landing techniques. Journal of Sports Science \& Medicine, 12/1, 122-129.

Ruddock, A.D., \& Winter, E.M. (2016). Jumping depends on impulse not power. Journal of Sports Sciences, 34/6, 584-585. Available from https://doi.org/10.1080/02640414. 2015.1064157 .

Smits-Engelsman, B., Smits, R., Oomen, J., \& Duysens, J. (2008). Strength training does not affect the accuracy of force gradation in an isometric force task in young men. International Journal of Sports Medicine, 29/1, 59-65. Available from https://doi.org/10.1055/s-2007-964999.

Standing, R.J., \& Maulder, P.S. (2015). A comparison of the habitual landing strategies from differing drop heights of parkour practitioners (traceurs) and recreationally trained individuals. Journal of Sports Science \& Medicine, 14/4, 723-731.
Toumi, H., Best, T.M., Martin, A., F'Guyer, S., \& Poumarat, G. (2004). Effects of eccentric phase velocity of plyometric training on the vertical jump. International Journal of Sports Medicine, 25/5, 391-8. Available from https://doi.org/ $10.1055 / \mathrm{s}-2004-815843$.

Turner, A.M., Owings, M., \& Schwane, J.A. (2003). Improvement in running economy after 6 weeks of plyometric training. Journal of Strength and Conditioning Research / National Strength \& Conditioning Association, 17/1, 60-7.

Wakai, M., \& Linthorne, N.P. (2005). Optimum take-off angle in the standing long jump. Human Movement Science, 24/1, 81-96. Available from https://doi.org/10.1016/j.humov. 2004.12.001.

Wu, W.-L., Wu, J.-H., Lin, H.-T., \& Wang, G.-J. (2003). Biomechanical analysis of the standing long jump. Biomedical Engineering: Applications, Basis and Communications, 15/5, 186-192. Available from https://doi.org/10.4015/ S1016237203000286.

Cite this article as: Grosprêtre S, Ufland P, \& Jecker D (2017) The adaptation to standing long jump distance in parkour is performed by the modulation of specific variables prior and during take-off. Mov Sport Sci/Sci Mot, 100, 27-37 